\title{
The origin of the mass scales for maximal star formation efficiency and quenching: the critical role of Supernovae
}

\author{
Bruno M. B. Henriques ${ }^{1 \star}$, Simon D. M. White ${ }^{2}$, Simon J. Lilly ${ }^{1}$, \\ Eric F. Bell ${ }^{3}$, Asa F. L. Bluck ${ }^{1}$, Bryan A. Terrazas ${ }^{3}$ \\ ${ }^{1}$ Department of Physics, ETH Zurich, CH-8093 Zurich, Switzerland \\ ${ }^{2}$ Max-Planck-Institut für Astrophysik, Karl-Schwarzschild-Str. 1, D-85741 Garching b. München, Germany \\ ${ }^{3}$ Department of Astronomy, University of Michigan, Ann Arbor, MI 48109, USA
}

Submitted to MNRAS, 2018

\begin{abstract}
We use the Henriques et al. (2015) version of the Munich galaxy formation model (LGALAXIES) to investigate why the halo and stellar mass scales above which galaxies are quenched are constant with redshift and coincide with the scale where baryons are most efficiently converted into stars. This model assumes that central galaxies are quenched by AGN feedback when hot halo gas accretes onto a supermassive black hole. Nevertheless, we find that supernova (SN) feedback sets both mass scales. As haloes grow above a threshold mass, SNe can no longer eject material so their hot gas content increases, enhancing the cooling rate onto the central galaxy, its cold gas content, its star formation rate and the growth rate of its central black hole. Strong AGN feedback terminates this short-lived phase by suppressing the fuel supply for star formation. Despite strong evolution of the halo mass - temperature relation, quenching occurs at a redshift-independent halo and stellar mass which coincides with the mass where baryons have been converted into stars with maximal efficiency. These regularities and coincidences are a result of the specific parameters selected by MCMC tuning of the model to fit the observed abundance and passive fraction of galaxies over the redshift range $0 \leqslant z \leqslant 3$. Thus they are required by the observed evolution of the galaxy population, at least in the context of models of this type.
\end{abstract}

Key words: galaxies: formation - galaxies: evolution - galaxies: high-redshift methods: analytical - methods: statistical

\section{INTRODUCTION}

One of the most striking features of the low-redshift galaxy population is that more than half of the stellar mass density is in quenched galaxies (Kauffmann et al. 2003; Baldry et al. 2004, Bell et al. 2004 Bundy et al. 2005 Faber et al. 2007). This value drops below $50 \%$ at $z=1$ and to $\sim 25 \%$ at $z=2$ (Ilbert et al. 2013, Muzzin et al. 2013 Tomczak et al. 2013). The likelihood of a galaxy being quenched has been found to correlate with a number of other galaxy (e.g., stellar mass, halo mass, black hole mass, central density) and environmental (e.g, central vs. satellite, halo mass, group centric radius) properties. Stellar mass has received particular attention, with massive galaxies found more likely to be quenched (Kauffmann et al. 2003 . Brinchmann et al. 2004

\footnotetext{
^ E-mail:brunohe@phys.ethz.ch
}

Baldry et al. 2004) and to be quenched earlier (Thomas et al. 2005 Bundy et al. 2005, Faber et al. 2007, Scarlata et al. 2007. Ilbert et al. 2013 Muzzin et al. 2013, Tomczak et al. 2013). Peng et al. (2010) showed that both trends are reproduced if the probability of a quenching event is assumed to increase strongly with stellar mass in a redshift-independent way (at least for $z \lesssim 1$ ).

Quenching the most massive galaxies requires some dramatic phenomena. Since the early work of White \& Frenk (1991) it has been clear that gas cooling rates in massive haloes will result in overly massive central galaxies unless they are substantially offset by some heating process; the development of static hot atmospheres and the reduced halo accretion rates in dark energy dominated cosmologies are insufficient on their own to explain why such a small fraction of the baryons associated with massive haloes lie in their central galaxies. Successful models for the formation of the galaxy population in a $\Lambda \mathrm{CDM}$ universe are forced to include

(C) 2018 RAS 
an additional feedback mechanism to suppress cooling of gas in such haloes, with AGN feedback being the preferred candidate (Springel et al. 2005a Croton et al. 2006, Bower et al. 2006). However, it remains unclear why feedback from hot gas accretion onto a central black hole results in such a well defined and redshift-independent stellar mass at quenching.

Another striking feature of the galaxy population is that the ratio between the stellar mass of a central galaxy and the mass of its halo shows a sharp peak at $\log _{10}\left(M_{200 \mathrm{c}} / \mathrm{M}_{\odot}\right) \sim$ 12.0 , corresponding to a maximum efficiency of $\sim 20 \%$ for converting baryons into stars (Guo et al. 2010, Moster et al. 2010). This efficiency falls off quite strongly for halo masses above and below this characteristic value which again appears to be almost independent of redshift (Moster et al. 2012, Behroozi et al. 2013). As highlighted by Birrer et al. (2014), and more recently by Rodriguez-Puebla et al. (2017), this scale of maximal efficiency seems to coincide with the mass above which galaxies are typically quenched. This coincidence and the invariance of this mass scale, at least since $z \sim 3$, seems particularly puzzling given the strong evolution in the typical density and temperature of halos of given mass.

In the Henriques et al. (2015) model, AGN feedback suppresses cooling and star formation in massive galaxies, resulting in a strong dependence between quenching and black hole mass as observed (Bluck et al. 2016. Terrazas et al. 2016b a, 2017), although the transition may be too abrupt in the model. In addition, Henriques et al. (2016) showed that this particular implementation of AGN feedback couples with environmental effects to reproduce quite well the trends with stellar mass and environment identified by Peng et al. (2010). Here we investigate why the interplay between the different feedback mechanisms in our model also reproduces the remarkably strong observed dependence of quenching and star formation efficiency on halo/stellar mass and the relative weak dependence of these relations on redshift. Of particular relevance is the emergence of this behaviour in a model where star formation strangulation from AGN feedback is a consequence of black hole growth without requiring a direct dependance with stellar or halo mass.

\section{THE MODEL}

The model used here is fully described in the supplementary material of Henriques et al. (2015 ${ }^{1}$ (hereafter H15). The evolution of the baryonic components - including hot, cold and ejected gas, stars in disks, bulges and haloes, and black holes - is self-consistently coupled to the evolution of dark matter subhaloes identified in dark-matter-only Nbody simulations. These are scaled to the Planck cosmology $\left(\sigma_{8}=0.829, H_{0}=67.3 \mathrm{~km} \mathrm{~s}^{-1} \mathrm{Mpc}^{-1}, \Omega_{\Lambda}=0.685\right.$, $\Omega_{\mathrm{m}}=0.315, \Omega_{\mathrm{b}}=0.0487, f_{\mathrm{b}}=0.155$ and $\left.n=0.96\right)$ according to the procedures of Angulo et al. (2014). Specifically we

1 The supplementary material is attached to the main paper at arXiv:1410.0365. Snapshot and light-cone catalogues are publicly available at: http://www.mpa-garching.mpg.de/millennium. The source code which generated the model is now also public at: http://galformod.mpa-garching.mpg.de/public/LGalaxies/ This page also contains catalogues in fits format and additional material related to the model. use the Millennium (Springel et al. 2005b) and MillenniumII (Boylan-Kolchin et al. 2009) simulations which each trace $2160^{3}$ ( $\sim 10$ billion) particles from $\mathrm{z}=127$ to the present day. The Millennium was carried out in a box of original side $500 \mathrm{~h}^{-1} \mathrm{Mpc}=685 \mathrm{Mpc}$. After rescaling to the Planck cosmology, this becomes $714 \mathrm{Mpc}$, implying a particle mass of $1.43 \times 10^{9} \mathrm{M}_{\odot}$. The Millennium-II follows a region one fifth the linear size, resulting in 125 times better mass resolution. Combined, the two simulations follow dark matter haloes which host galaxies spanning five orders of magnitude in stellar mass at $z=0$. Throughout the paper, the Millennium-II Simulation is used for $\log _{10}\left(M_{*} / \mathrm{M}_{\odot}\right) \leqslant 10.0$ and $\log _{10}\left(M_{200 c} / \mathrm{M}_{\odot}\right) \leqslant 11$ and the Millennium Simulation for higher stellar masses. Above this mass cut, properties of galaxies are nearly identical in the two simulations. The exception are properties that strongly depend on merger histories for which we restrict our analysis to MRII (this is the case in Fig 7).

The self-consistent treatment of the evolution of the stellar, gas and black hole components of galaxies assumes that baryons and dark matter are initially fully mixed and that collapsed dark matter structures have a baryon fraction given by the cosmic mean for the adopted cosmology. Baryons are initially assumed to be in the form of diffuse hot gas which either cools immediately or forms a static hot atmosphere with a central cooling flow. Cold gas settles in a disk and fuels star formation which eventually leads to the release of energy and metals through SN. This can either reheat gas back into the hot halo phase or eject it entirely into an external reservoir from which it might be subsequently reincorporated. Mergers result in starbursts and drive the growth of both bulges and central black holes through cold gas accretion. Subsequent hot gas accretion onto black holes generates AGN "radio-mode" feedback which can completely shut down cooling. Whenever galaxies become satellites, and before they merge with their central companions, environmental effects gradually remove their hot, cold and stellar components.

Of particular relevance for the present study is our adopted implementation of SN feedback. Following Guo et al. (2011) the efficiencies of reheating (of cold gas into the hot phase) and ejection (of hot gas into the external reservoir) are assumed to scale inversely with the virial velocity of the halo, while the timescale for reincorporation of ejected gas scales inversely with virial mass (Henriques et al. 2013). Also important are our black hole growth and AGN feedback models. Black hole growth is dominated by cold gas accretion during mergers which is assumed to be more efficient in more massive systems (Kauffmann \& Haehnelt 2000 ) while their feedback is determined by hot gas accretion. The energy output is assumed to scale as the product of the black hole and hot gas masses, and is used to offset the cooling of hot gas (Croton et al. 2006; H15).

In order to better understand the impact of different feedback channels in the conversion of baryons into stars and in star formation quenching, throughout the paper we will analyse the results from four variations of the H15 model: the original model, a model with no AGN and no SN feedback (no-feedback model), a model without SN feedback (AGN-only model) and a model without AGN feedback (SNonly model). For all model variations the parameters are kept constant at the H15 values, set by the MCMC sam- 


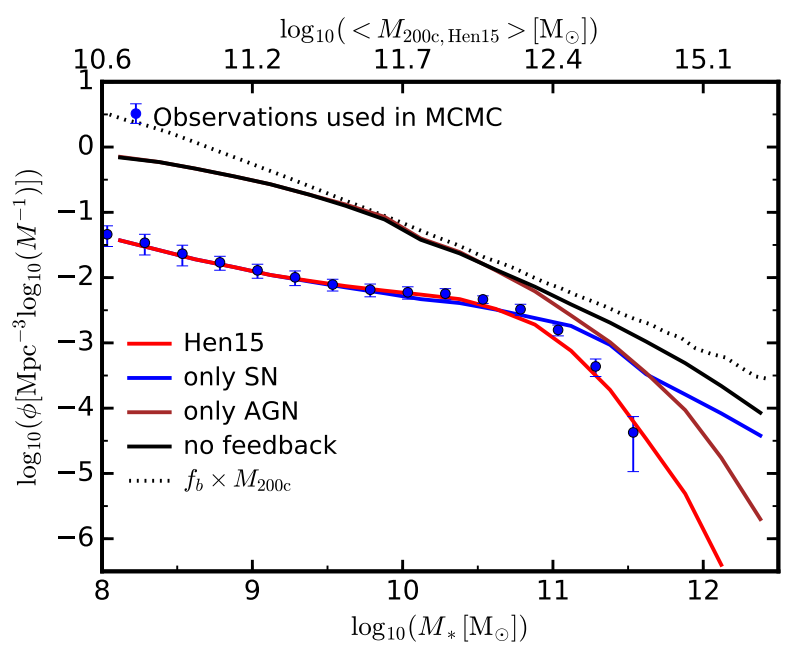

Figure 1. Stellar mass functions at $z=0$ for different variations of the H15 model (shown as a solid red line). The solid black, blue and brown lines show, respectively, versions of the H15 model with neither AGN nor SN feedback, with SN feedback only (no AGN) and with AGN feedback only (no SN). The dotted black line shows the halo mass function scaled by the baryon fraction. The halo mass scale on the upper $x$-axis applies to the full H15 model only. The Millennium-II Simulation is used for $\log _{10}\left(M_{*} / \mathrm{M}_{\odot}\right) \leqslant 10.0$ and the Millennium Simulation for higher stellar masses.

pling constrained by the observed abundance and passive fraction of galaxies over the redshift range $0 \leqslant z \leqslant 3$, which makes it easier to isolate the impact of different feedback channels.

\section{RESULTS}

In our self-consistent model for the evolution of galaxies, energy feedback from SN and AGN competes with accretion, cooling and star formation to establish the rates for conversion of baryons into cold gas and stars. We will start our analysis by trying to understand how these different mechanisms combine to produce, at $z=0$, a characteristic stellar mass above which galaxies tend to be quenched. We also investigate why this quenching threshold coincides with the halo mass at which the integrated efficiency of converting baryons into stars is maximal. We will then investigate why this characteristic mass scale remains constant out to at least $z \sim 3$.

\subsection{The stellar mass function at $z=0$ : a mass scale for peak efficiency of converting baryons to stars}

In Fig. 1 we show stellar mass functions at $\mathrm{z}=0$ for the four models used in our study: the standard H15 model (solid red line), the no-feedback model (solid black line), the AGN-only model (solid brown line) and the SN-only model (Solid blue line). In addition, the black dotted line shows the halo mass function multiplied by the cosmic fraction of baryons in our cosmology (0.155). The top x-axis shows the average $M_{200 \mathrm{c}}$ for a given stellar mass in the H15 model (note that this does not correspond to the mean halo mass at this stellar mass in the other three models). We see that without any form of feedback (solid black line) most of the baryons are converted into stars and the stellar mass function closely follows the re-scaled halo mass function (dotted black line). The exceptions are the very low- and high-mass ends where, respectively, photo-heating by the UV background and the development of a hot atmosphere make cooling less efficient. In addition, at high-mass a significant fraction of the baryons are in satellite galaxies and the intracluster light.

When AGN feedback alone is included (solid brown line) the high-mass tail of the stellar mass function is significantly reduced above $\log _{10}\left(M_{*} / \mathrm{M}_{\odot}\right) \sim 11$, corresponding in this model to $\log _{10}\left(M_{200 \mathrm{c}} / \mathrm{M}_{\odot}\right) \sim 12.2$, while the low-mass end remains unchanged. The onset of AGN feedback and the suppression of cooling and galaxy growth happens at the observed stellar mass scale, coinciding with the formation of a substantial hot halo. This is a consequence of the assumed relation between the strength of radio mode feedback and hot gas mass. However, as we will see in section 3.3 without SN winds this mass scale evolves too strongly with redshift. In addition, the reduction in the stellar content of galaxies inhabiting the most massive haloes is much less pronounced than in observations.

More dramatic changes arise when SN feedback is included (solid blue line): there is a large reduction in the stellar mass function at all masses with respect to the no-feedback case, but particularly for low-mass galaxies. A clear characteristic mass appears, again at about $\log _{10}\left(M_{*} / \mathrm{M}_{\odot}\right) \sim 11$ corresponding in this case to $\log _{10}\left(M_{200 \mathrm{c}} / \mathrm{M}_{\odot}\right) \sim 12.4$, below which the reduction is more significant. The reduction at high masses with respect to the no-feedback case is caused both by the reheating of cold gas in massive galaxies (despite a failure to eject any hot gas) and by the reduced stellar masses of accreted satellites.

Once both feedback channels are included (solid red line) suppression is effective at both low and high masses with the knee (where the stellar mass function is closest to the scaled halo mass function) being shifted to slightly lower mass than in the SN-only case. This first plot clearly shows that, while SN feedback is solely responsible for the reduction in the number of low-mass galaxies, both $\mathrm{SN}$ and AGN feedback are required to explain the sharp cutoff at the high-mass end. The combination of the two effects results in a relatively small range in stellar mass for which the fraction of baryons converted into stars is maximal.

\subsection{The stellar mass/halo mass relation at $z=0$ : a quenching threshold that coincides with maximal star-formation efficiency}

The stellar mass functions shown in the previous subsection seem to indicate that the interplay between SN and AGN feedback plays a critical role in reducing the range in stellar mass for which the fraction of baryons converted into stars is close to maximal and in establishing a sharp high-mass cutoff. Here we investigate this further by looking at the relation between stellar-to-halo mass ratio and halo mass as a function of star formation activity in the central galaxy. This will help us to understand why the scale of maximal integrated star formation efficiency coincides with the threshold for quenching. 


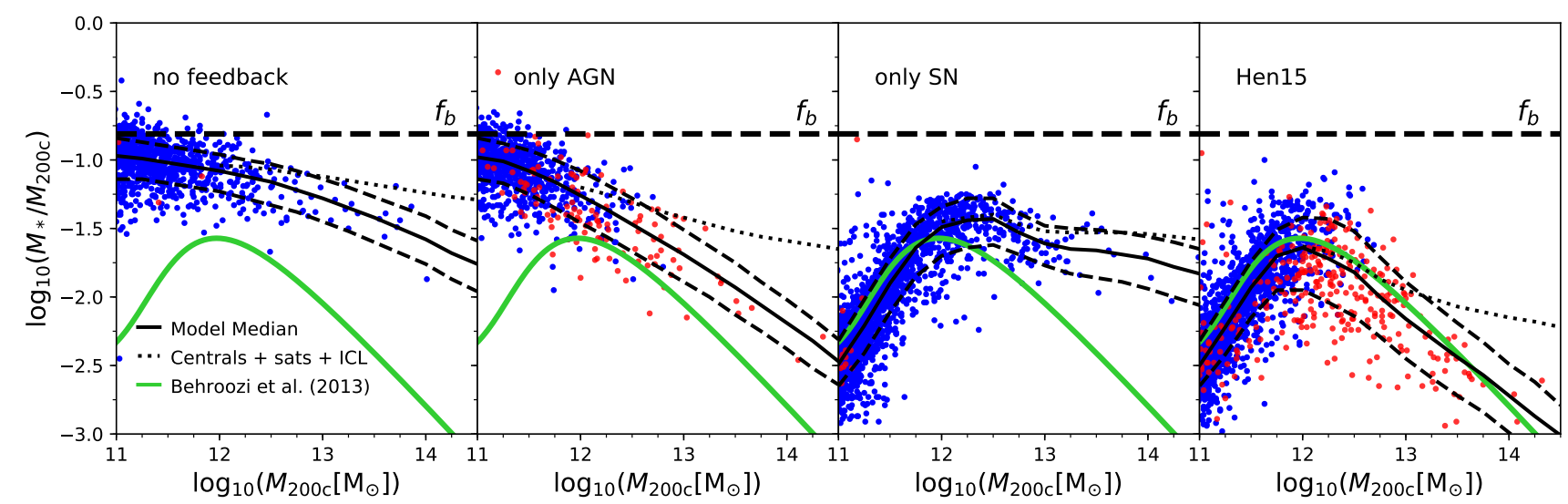

Figure 2. The stellar-to-halo mass ratio versus halo mass for central galaxies in the four models analysed in the present study: no feedback (left panel), AGN feedback only (middle-left panel), SN feedback only (middle-right panel) and the standard H15 model (right panel). The coloured dots show a random sample of galaxies in each model, with blue being star-forming and red being passive objects (divided at $\left.\log _{10}\left(s S F R\left[\mathrm{yr}^{-1}\right]\right)=-11.0\right)$. The solid and dashed black lines show, respectively, the median and $1 \sigma$ scatter around the median for the different models, while the solid green line shows an abundance matching estimate from Behroozi et al. (2013). The dotted black line shows the model median including satellites and ICL. A clear peak in the distribution appears when SN feedback is included (middle-right panel) which coincides with the mass scales at which galaxies transition from star-forming to quenched when AGN feedback is present (right panel).

Fig. 2 plots the ratio of central galaxy stellar mass to halo mass against halo mass for our four models: no-feedback (left panel), AGN-only (middle-left panel), SN-only (middleright panel) and the standard H15 model (right panel). Without feedback almost all baryons are turned into stars in low-mass halos. At high mass, the formation of a static hot halo delays cooling and reduces star formation. In addition, in this mass range, a significant fraction of the baryons are in satellite galaxies or in the intracluster light (as shown by the dotted black line). However, as seen for the stellar mass function, this is insufficient to explain the very large reduction in baryon conversion efficiency in massive haloes derived from abundance matching (e.g. Behroozi et al. 2013 the solid green line). It is also unable to explain why massive galaxies are predominately quenched since these continue to form stars despite the delayed cooling.

When AGN feedback alone is included (second panel from the left) the results are similar at low halo mass, but the star-formation efficiency is much lower at high mass. As explained in Section 4.2, radio-mode feedback is proportional to black hole and hot gas mass in the H15 model. Quenched galaxies appear and are spread relatively evenly (by number) across halo mass, and they predominate at high halo mass $\left(\log _{10}\left(M_{200 c} / \mathrm{M}_{\odot}\right)>12.5\right)$ as in the full model. However, without SN feedback, galaxies are extremely gas-rich, and large black holes and overly massive stellar populations can form even in low-mass haloes. Large reservoirs of cold gas survive because AGN feedback is not fueled by, and has no effect on cold gas in the H15 model. This results in a relatively small fraction of quenched galaxies in lower mass haloes.

In contrast, for the SN-only case (second panel from the right), a peak appears in the stellar-to-halo mass ratio. In the context of our model, at low masses the ejection of hot gas into the external reservoir greatly suppresses star formation. This ceases to be effective at $\log _{10}\left(M_{200 c} / \mathrm{M}_{\odot}\right) \sim$
12 where the potential wells become too deep for ejection to be possible. At higher mass the conversion efficiency is still suppressed with respect to the no-feedback case because cooling from the hot atmosphere is partially offset by the return of disk gas reheated by $\mathrm{SN}$ and also because accreting satellites have fewer stars. Since SN regulate the supply of cold gas but are unable to eliminate it completely, there are no quenched central galaxies in this case. In our model, this only happens as a result of AGN radio mode feedback from massive black holes. As detailed below, SN are critical in establishing the halo/stellar mass scale at which black holes can grow and subsequently release energy, but are not directly responsible for the quenching due to their inability to stop the hot gas atmosphere from cooling.

In the full H15 model where both SN and AGN feedback are included (right panel), the peak in conversion efficiency created by SN feedback remains. However, a clear threshold for quenching by AGN feedback emerges at the same mass. The fraction of baryons in cold gas is relatively low in lowmass galaxies (with respect to their total baryon content) and their assembly histories typically involve fewer of the equal-mass galaxy mergers required for black hole growth in the $\mathrm{H} 15$ model ${ }^{2}$ As a result, the ratio of black hole to stellar mass remains much lower than in more massive systems. Furthermore, efficient cooling leaves rather little hot gas, so AGN feedback is negligible. At the halo mass where ejection ceases to be effective, the hot halos become more massive and cooling from them causes the central galaxies to grow large cold gas reservoirs. As will become clearer in the following sections, this leads not only to maximally efficient star formation but also to increased black hole growth. Shortly thereafter, the black holes become massive enough for AGN feedback to suppress cooling altogether, thus sup-

2 Note that Bell et al. (2017) argue that observationally it is unclear which mergers are associated with black hole growth. 


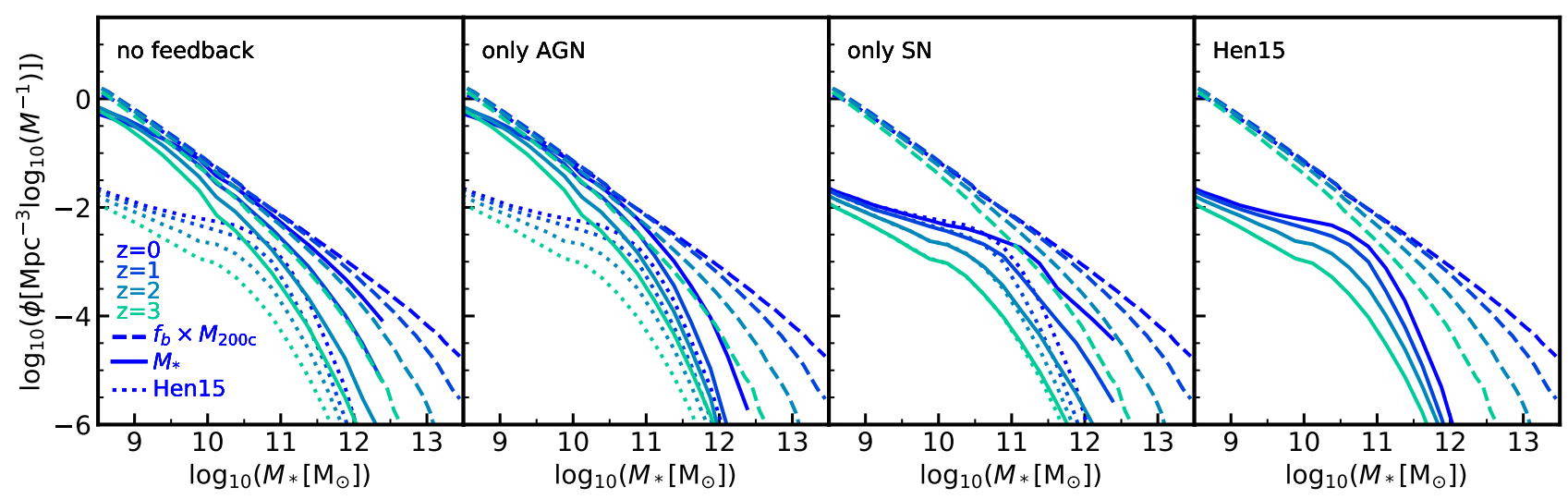

Figure 3. The evolution from $z=3$ to $z=0$ of the stellar mass function (solid coloured lines) and of the halo mass function multiplied by the cosmic fraction of baryons (dashed coloured lines). From left to right, the different panels represent the no-feedback model, the AGN-only model, the SN-only model and the standard H15 model. In the three left panels, the coloured dotted lines show the evolution of the stellar mass function in the standard H15 model. The Millennium-II Simulation is used for $\log _{10}\left(M_{*} / \mathrm{M}_{\odot}\right) \leqslant 10.0$ and the Millennium Simulation for higher stellar masses (this explains the small discontinuity visible in the transition between the two).

pressing star formation. This is the apparent coincidence highlighted in the data by Birrer et al. (2014).

In the H15 model, the relation between the efficiency of hot gas ejection by $\mathrm{SN}$ and $M_{200 \mathrm{c}}$ is controlled by parameters which the MCMC sampling sets primarily in order to fit the observed stellar mass function at low mass. The detailed dependence results in SN being unable to eject gas from haloes more massive than $\log _{10}\left(M_{200 \mathrm{c}} / \mathrm{M}_{\odot}\right) \sim 12$.

\subsection{The evolution of the stellar mass function: a constant scale for peak efficiency of converting baryons to stars}

Henriques et al. (2016) already gave indications that the stellar mass threshold for quenching and the stellar mass at which the integrated efficiency of star formation peaks are similar, and that both are constant with redshift. The constancy of the former was suggested by the weak evolution of the "knee" of the stellar mass function for star-forming galaxies, while that of the latter was suggested by the similarly constant knee in the stellar mass function for all galaxies. Here we look in more detail at the evolution of the total stellar mass function in order to better understand why the stellar mass at the knee is constant despite the fact that the characteristics of the corresponding haloes are evolving strongly.

In Fig. 3 we plot the $z=3$ to $z=0$ evolution of the stellar mass function for all galaxies as solid coloured lines, and that of the halo mass function multiplied by the cosmic baryon fraction as dashed coloured lines. From left to right, the different panels represent the no-feedback model, the AGN-only model, the SN-only model and the standard H15 model. In the three left panels, the coloured dotted lines reproduce the stellar mass functions from the standard H15 model. We see that, except for the full H15 model (right panel), all stellar mass functions appear to show an evolving characteristic mass. Without any feedback (left panel), the stellar mass functions at low mass correspond to almost $100 \%$ conversion into stars of the available baryons.
At higher mass the conversion is less efficient and the solid curves fall progressively to the left of the dashed ones. With decreasing redshift this reduction below $100 \%$ efficiency occurs at larger and larger stellar (and hence halo) mass.

The introduction of AGN feedback (second panel from the left) has very little effect on the stellar mass functions at low mass $\left(\log _{10}\left(M_{*} / M_{\odot}\right)<10.5\right)$ or high redshift $(z \sim 3)$ but at lower redshift the stellar masses of the central galaxies in high-mass haloes are reduced, shifting the solid curves further to the left. The evolution of the characteristic mass is reduced, although not as much as in the H15 model (and not to the observed level). In contrast, in the SN-only case (second panel from the right) the stellar mass functions are reduced substantially and deviations from the full H15 model are seen only at high mass and low redshift. At $z=3$ the stellar mass function of the SN-only model is essentially identical to that of the full model. Nevertheless, the characteristic mass evolves as in the no-feedback model. In the final H15 model (right panel), AGN feedback suppresses cooling and star formation in the most massive halos at later times. This effectively eliminates the progression of the knee of the stellar mass function towards higher masses which is seen in the SN-only model, resulting in an almost constant characteristic mass, as required by the observations $\left(\log _{10}\left(M_{*} / M_{\odot}\right) \sim 11.4,10.9,11.1\right.$ and 10.7 , respectively at $z=3,2,1$ and 0 , see Fig. 2 of Henriques et al. 2016). In summary, AGN and SN feedback alone can create a characteristic mass, this remains constant at $z \leqslant 3$ when both are combined. In this case, as we will see in the next section, black holes can only grow from cold gas accretion above a very weakly evolving halo mass scale at which SN winds become inefficient.

\section{THE ORIGIN OF THE CHARACTERISTIC MASS SCALE}

Previous sections identified SN feedback as a necessary ingredient in establishing a relatively narrow and weakly 
evolving mass scale at which the fraction of baryons converted into stars maximizes. Although AGN quenching reduces the evolution of the characteristic mass of the stellar mass function, it only suppresses it completely when combined with SN. In addition, when SN are present, AGN produce a relatively sharp transition between haloes hosting star-forming and passive galaxies, that coincides with maximal efficiency of baryon conversion into stars. In this section we will investigate how individual physical processes interact to produce this behaviour.

For reasons illustrated in Figures 4 and 5 we find it useful to distinguish three different halo mass regimes, corresponding roughly to $\log _{10}\left(M_{200 \mathrm{c}} / \mathrm{M}_{\odot}\right)<12$, $\log _{10}\left(M_{200 \mathrm{c}} / \mathrm{M}_{\odot}\right) \sim 12$ and $\log _{10}\left(M_{200 \mathrm{c}} / \mathrm{M}_{\odot}\right)>12$. These figures plot the median and 16 to $84 \%$ scatter in the fractions of the total baryons associated with five different components: the stars, the cold gas and the supermassive black hole associated with the central galaxy, together with the hot gas associated with the surrounding main subhalo, and the material stored in the reservoir of halo ejecta (this represents material ejected by SN winds, which might be reincorporated at later times into the hot gas). Fig. 4 shows these fractions as a function of halo mass at $z=0$, while Fig. 5 shows them in the form of the masses of the individual components (and also of the halo itself) as a function of redshift for halos which at $z=0$ have masses $\log _{10}\left(M_{200 c} / \mathrm{M}_{\odot}\right) \sim 11,12$, 13 and 14 . In both figures the grey shaded regions mark the transition phase between when the hot component becomes more massive than the external reservoir (when reincorporation balances SN ejection) and when star formation is quenched $\left(s S F R<(1+z) / 2 t_{\mathrm{H}}\right.$ is used to select passive galaxies in the full model at different redshifts following Fig. 9 of Terrazas et al. 2016b), while the vertical dashed lines mark the peak of star formation activity.

For low-mass haloes where cooling times are short, infalling material shocks, cools and condenses as it hits the galaxy, thus immediately becoming available to form stars. Since the SN ejection of hot gas into the external reservoir scales inversely with $V_{\max }$ and gas reincorporation timescales are long, most newly accreted gas is ejected from the halo by SN winds and does not return. The baryons associated with such haloes are therefore predominantly in the external reservoir, as can be seen in the purple line in Fig. 4 at $\log _{10}\left(M_{200 c} / \mathrm{M}_{\odot}\right)<12$. As $\log _{10}\left(M_{200 \mathrm{c}} / \mathrm{M}_{\odot}\right)$ approaches $\sim 12$, SN cease to be effective at expelling gas, and the baryon fraction in the external reservoir drops dramatically (low mass boundary of grey shaded region). At the same time, hot gas cooling times lengthen to the point that a substantial hot component can be maintained, and infalling gas adds directly to this atmosphere (red line). However, cooling is still significant, leading to a peak both in the cold gas content (blue line) and in the specific star-formation rate (vertical dashed line). Since our model assumes central black holes to be built up by merger-driven accretion of cold gas, they grow rapidly in this regime (brown line) and soon reach masses for which AGN feedback provides enough energy to halt further cooling from the hot gas halo (high mass boundary of grey shaded region). At higher mass, $z=0$ halos have little cold gas, no external reservoir, and a declining baryon fraction in the central stellar component. Interestingly, the model predicts central black hole mass to be proportional to

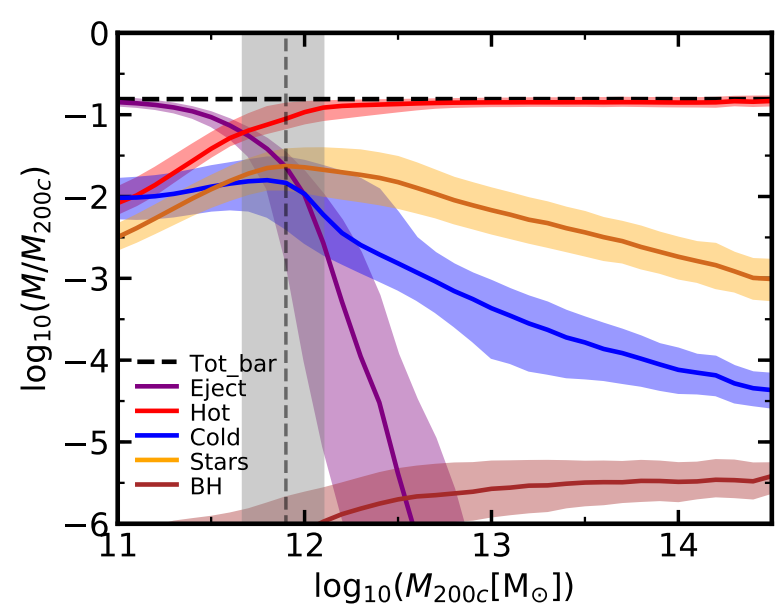

Figure 4. Mass fraction in different components with respect to the virial mass as a function of the latter for central galaxies. The solid lines represent the median and the coloured regions the 16th to 84th percentiles of the distributions for $M_{\text {eject }} / M_{200 c}$ (purple), $M_{\text {hot }} / M_{200 c}$ (red), $M_{\text {cold }} / M_{200 c}$ (blue), $M_{*} / M_{200 c}$ (orange) and $M_{\mathrm{BH}} / M_{\mathrm{vir}}$ (brown). The dashed black line represents the median $M_{\text {baryon }} / M_{200 c}$. The grey shaded region shows the halo mass between when the hot component becomes more massive than the external reservoir (when reincorporation balances SN ejection) and when star formation is quenched. The vertical dashed line marks the peak of star formation activity.

halo mass rather than to central galaxy stellar mass in this regime (cf Bogdan et al. 2017).

Fig. 5 shows that at all redshifts, low-mass halos are in the same regime as at $z=0$ (left panel), while high-mass halos enter the transition region between the two asymptotic regimes at the redshift when their progenitors have $\log _{10}\left(M_{200 \mathrm{c}} / \mathrm{M}_{\odot}\right)$ just below $12(z \sim 6, z \sim 4$ and $z \sim 2$, respectively in the first, second and third panels from the right).

\subsection{The SN feedback dominated and intermediate regimes: $M_{200 \mathrm{c}} \leqslant 10^{12} \mathrm{M}_{\odot}$}

The behaviour of our model in the low-mass regime can be derived simply from our underlying assumptions about cooling and the effects of SN feedback. These are set out in detail in the supplementary material (SM) to Henriques et al. (2015) which can be found online attached to the arXiv preprint. The haloes of low-mass galaxies are always in the rapid accretion regime where the bulk of newly accreted baryons are assumed to fall onto the central galaxy and become available for star formation on a short time-scale (see Section S1.4 of the SM). Because such haloes have shallow potential wells, most newly accreted material is immediately ejected from the halo in a SN-driven wind and the amount of material turned into new stars is just that needed to provide the requisite ejection energy.

The energy input into outflowing gas is assumed to be directly proportional to the mass of stars formed,

$$
\Delta E_{\mathrm{SN}}=\epsilon_{\text {halo }} \times \frac{1}{2} \Delta M_{*} V_{\mathrm{SN}}^{2},
$$

where $V_{\mathrm{SN}}=630 \mathrm{~km} / \mathrm{s}$ for the IMF we assume, and the 


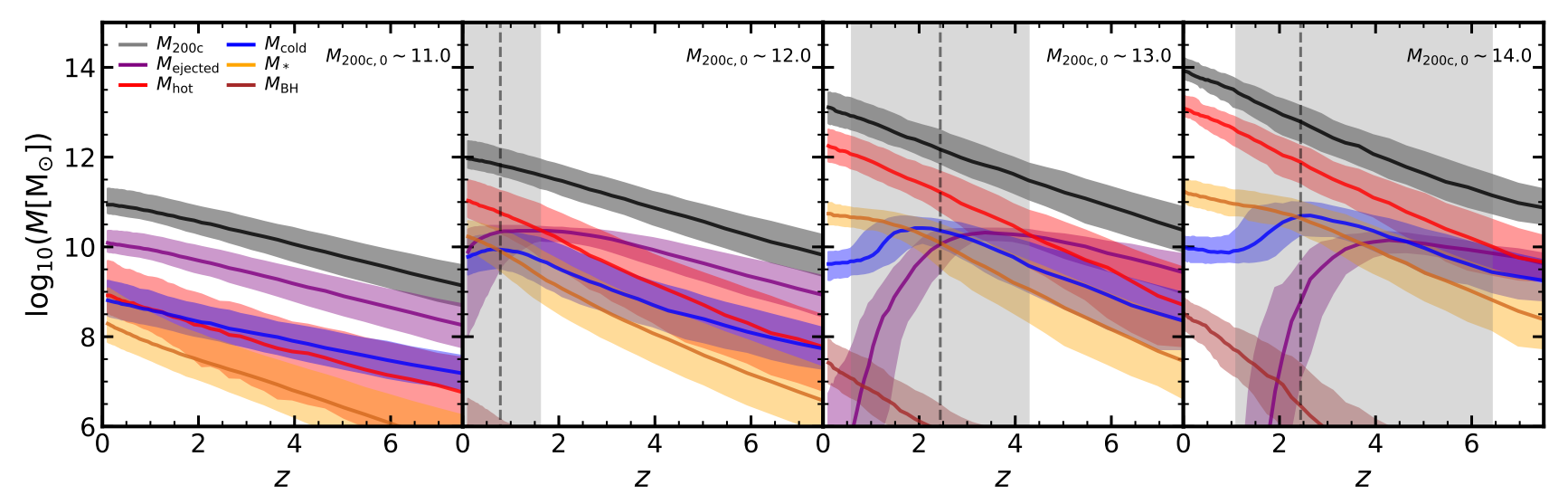

Figure 5. The evolution of the mass in different components in the H15 model for objects which at $z=0$ lie at the centre of haloes with virial mass $10^{11} \mathrm{M}_{\odot}$ (left panel), $10^{12} \mathrm{M}_{\odot}$ (centre left panel), $10^{13} \mathrm{M}_{\odot}$ (centre right panel) and $10^{14} \mathrm{M}_{\odot}$ (right panel). The solid lines represent the median and the coloured regions the 16th to 84th percentiles of the distribution for $M_{200 c}$ (black), $M_{\text {eject }}$ (purple), $M_{\text {hot }}$ (red), $M_{\text {cold }}$ (blue), $M_{*}$ (orange) and $M_{\mathrm{BH}}$ (brown). The grey shaded region in the three right panels highlights the redshift range between the time when the hot component becomes more massive than the external reservoir and that when star formation is quenched. The vertical dashed lines mark the peak of star formation activity. The Millennium-II Simulation is used in all panels except the right-most in order to correctly follow the very low mass progenitors of the haloes considered.

efficiency $\epsilon_{\text {halo }}$ decreases with increasing $V_{200 c}$, is unity for haloes with $V_{200 c} \sim 100 \mathrm{~km} / \mathrm{s}$ and drops to about 0.4 for large $V_{200 c}$ (equation S17 of the SM). Some of this energy is used to reheat a mass:

$$
\Delta M_{\text {reheat }}=\epsilon_{\text {disk }} \Delta M_{*}
$$

from the cold ISM to the hot halo, while the rest is used to drive gas out of the hot halo into the external reservoir. The mass loading factor $\epsilon_{\text {disk }}$ varies with disk rotation velocity as $8 \times\left(V_{\text {rot }} / 100 \mathrm{~km} / \mathrm{s}\right)^{-0.72}$ over the $V_{\text {rot }}$ range of relevance (equation S19 of the SM). A specific energy $\frac{1}{2} V_{200 c}^{2}$ per unit mass is assumed to be necessary for both the cold gas reheating and hot gas ejection processes resulting in:

$$
\Delta M_{\text {eject }}=\left(\epsilon_{\text {halo }} V_{\mathrm{SN}}^{2} / V_{200 c}^{2}-\epsilon_{\text {disk }}\right) \Delta M_{*} .
$$

The material ejected from the hot halo is subsequently reincorporated at a rate given by:

$$
\dot{M}_{\text {ejected }}=-\frac{M_{\text {eject }}}{t_{\text {reinc }}}
$$

where $t_{\text {reinc }}=\gamma 10^{10} / M_{200 \mathrm{c}}$ and $\gamma=3 \times 10^{10} \mathrm{yr}$.

Assuming that the baryonic mass newly accreted onto the halo is $f_{b} \Delta M_{200 c}$, where $f_{b}$ is the cosmic baryon fraction, that the sum of the mass changes in the cold and hot gas components is small enough to be neglected and that reincorporation times are long enough to prevent any significant amount of material to return from the external reservoir, we can write:

$$
f_{b} \Delta M_{200 c} \approx \Delta M_{\text {eject }}+\Delta M_{*}
$$

This is the case for small haloes where the first term in the parenthesis on the rhs of equation 3 is also dominant and $\epsilon_{\text {halo }}=1$, giving:

$$
f_{b} \Delta M_{200 c} \approx \Delta M_{\text {eject }} \approx\left(V_{\mathrm{SN}}^{2} / V_{200 c}^{2}\right) \Delta M_{*},
$$

which, if haloes build up the bulk of their mass at approxi- mately constant $V_{200 c}$, implies

$$
M_{*} / M_{200 c} \propto V_{200 c}^{2} \propto\left(G M_{200 c} H(z)\right)^{2 / 3},
$$

where $H(z)$ is the Hubble constant as a function of redshift. This behaviour can be seen at low masses in Fig. 4 (orange line). It is also visible by comparing the four panels of Fig. 5 where both the stellar mass and the redshift of the point where the median progenitor halo mass is $10^{11} \mathrm{M}_{\odot}$ increase with increasing final halo mass (the value of the orange line when the black line reaches $\left.10^{11} \mathrm{M}_{\odot}\right)$.

As the virial velocity $V_{200 c}$ of halos increases, the value of the parenthesis on the rhs of equation 3 drops, reaching zero at $V_{200 c} \sim 200 \mathrm{~km} / \mathrm{s}$ for the specific parameters of our model. This corresponds to $\log _{10}\left(M_{200 c} / \mathrm{M}_{\odot}\right) \sim 11.6$, $11.8,12.0$ and 12.3 at $z=3,2,1$ and 0 , respectively. In addition, the term in the bottom of equation 4 decreases and a significant amount of mass starts returning from the external reservoir. The reincorporation time becomes smaller than $0.1 \times t_{\text {dyn }}$ for halo masses larger than $\log _{10}\left(M_{200 c} / \mathrm{M}_{\odot}\right) \sim 12.2,11.9,11.7$ and 11.5 at $z=3,2,1$ and 0 , respectively, meaning that any ejected material is almost immediately reincorporated. The combined effect of reduced ejection into, and increased reincorporation from, the external reservoir creates a characteristic mass, $M_{200 c}^{\prime}$, above which SN are unable to maintain gas outside haloes. This mass is almost independent of redshift (compare the high redshift boundary of the grey shaded regions in the three right panels of Fig. 5 just below $\left.\log _{10}\left(M_{200 \mathrm{c}} / \mathrm{M}_{\odot}\right) \sim 12\right)$.

Shortly after haloes reach this weakly evolving mass scale, there is a precipitous drop in the baryon fraction in the external reservoir and a corresponding increase in the amount of hot gas, which is the dominant baryonic component at $z=0$ in halos with $M_{200 c}>5 \times 10^{11} \mathrm{M}_{\odot}$ (see purple and red lines in Fig. 4 the increased fraction of hot gas is not as clear in Fig. 5 due to the many orders of magnitude plotted in the y-axis). The three right panels of Fig. 5 show that this transition occurs at $z \sim 2, z \sim 4$ and $z \sim 6$ for the main progenitors of haloes with present-day 
Bruno M. B. Henriques et al.

mass $\log _{10}\left(M_{200 c} / \mathrm{M}_{\odot}\right) \sim 12,13$ and 14, respectively. As expected, this corresponds to characteristic masses at that time which are similar to the present-day transition value.

In our model, this mass roughly corresponds to the virial temperature at which haloes switch between the rapid cooling and cooling flow regimes. Again, this is a consequence of the particular parameter values picked out by our MCMC chains and is required in order for a substantial fraction of the baryons to be able to remain in the hot phase for an extended period. Nevertheless, cooling rates are still quite large at the transition point, resulting in peaks in cold gas fraction and specific star formation rate soon after haloes pass through this transition (vertical dashed lines in the three right panels of Fig. 5). This causes an acceleration in black hole growth, and shortly thereafter the cold gas fraction drops dramatically as the majority of galaxies quench (the low redshift boundary of the grey shaded regions at $z \sim 1.0$ and $z \sim 0.5$ in the $\log _{10}\left(M_{200 \mathrm{c} / \mathrm{M}_{\odot}}\right) \sim 14$ and 13 cases, respectively the first and second panels from the right in Fig. 5). Although cooling times lengthen in these more massive haloes as they grow, these sharp drops in cold gas fractions require an additional quenching process. In our model this is AGN feedback, as we discuss next.

\subsection{The AGN quenching regime}

The previous subsection detailed how our SN feedback model leads to a peak in cold gas fraction and hence to enhanced black hole growth at $\log _{10}\left(M_{200 \mathrm{c}} / \mathrm{M}_{\odot}\right) \sim 12$ where the proportion of hot gas is also rapidly increasing. We now explore why this results in AGN triggering and the quenching of star formation at this same mass scale. In order to better understand the quenching process, we will look at the balance between AGN heating and cooling of hot halo gas as a function of stellar mass. In the H15 model, black holes are assumed to grow predominately via cold gas accretion during galaxy mergers:

$$
\Delta M_{\mathrm{BH}, \mathrm{Q}}=\frac{f_{\mathrm{BH}}\left(M_{\mathrm{sat}} / M_{\mathrm{cen}}\right) M_{\mathrm{cold}}}{1+\left(V_{\mathrm{BH}} / V_{200 \mathrm{c}}\right)^{2}},
$$

while AGN heating, $\dot{E}_{\text {radio }}=\eta \dot{M}_{\mathrm{BH}} c^{2}$, is assumed to be proportional to the product of black hole mass and hot gas mass:

$$
\dot{M}_{\mathrm{BH}}=k_{\mathrm{AGN}}\left(\frac{M_{\mathrm{hot}}}{10^{11} \mathrm{M}_{\odot}}\right)\left(\frac{M_{\mathrm{BH}}}{10^{8} \mathrm{M}_{\odot}}\right)
$$

and $f_{\mathrm{BH}}, V_{\mathrm{BH}}$ and $k_{\mathrm{AGN}}$ are tuneable parameters.

As halo masses approach $10^{12} \mathrm{M}_{\odot}$, hot gas fractions and $\mathrm{BH}$ masses both grow rapidly, leading to a dramatic increase in feedback from AGN. For the parameters selected by our MCMC chains, this results in the complete suppression of cooling above a relatively narrow range in halo masses only slightly above the maximum for which SN feedback is able to maintain gas outside of haloes. We have found that this behaviour is still present in models without the $V_{200 c}$ dependence in equation 8 and/or without the $M_{\text {hot }}$ dependence in equation 9 It is important to highlight that our AGN feedback model only shuts down cooling without ejecting any gas from the halo. The subsequent quenching of star formation happens due to starvation after all the remaining cold gas is turned into stars (a similar behavour was inferred from the metal content of observed quenched galaxies in Peng et al. 2015).

Fig. 6 compares the balance between AGN heating and hot gas cooling as a function of the stellar mass of the central galaxy for two different models: AGN-only (on the left) and the full H15 model (on the right). The coloured dots show the values for individual galaxies at $z=0$ with red symbols representing quenched galaxies and blue symbols representing star-forming objects (divided at $\log _{10}\left(s S F R\left[\mathrm{yr}^{-1}\right]\right)=$ $-11.0)$. Note that in order to enhance the legibility of the plot, we here represent the heating rate by the value from equation 9, whereas in the model it is assumed to saturate when it equals the cooling rate. The dashed black line separates galaxies where cooling is fully suppressed (above) from galaxies where it is still occurring (below). The solid contours on the right panel show the $2 \mathrm{D}$ distribution of galaxies at different redshifts, while the vertical lines and solid bars at the bottom of this panel represent the median and the $16 \%$ to $84 \%$ range of stellar mass for galaxies that just crossed the black dotted line upwards (i.e. were just quenched). From top to bottom the bars refer to redshifts $\mathrm{z}=0,1,2$ and 3 . The top $x$-axis shows the average $M_{200 \mathrm{c}}$ for each stellar mass at $z=0$. Note that this axis differs between the two panels. Since galaxies with $\log _{10}\left(M_{*} / \mathrm{M}_{\odot}\right) \sim 9$ are hosted by haloes with very low mass in the AGN-only model we only show results based on the Millennium-II simulation for this plot.

Without SN feedback (left panel) we see that galaxies of a given stellar mass lie in substantially lower mass haloes than in the H15 model, and that in almost all haloes with $\log _{10}\left(M_{200 \mathrm{c}} / \mathrm{M}_{\odot}\right) \gtrsim 10.5$ the cooling from the hot gas halo is fully suppressed. This is in part because they have, on average, more massive black holes (which increases the heating rate) and in part because they have lower amounts of hot gas (which decreases the cooling rate more than the heating rate). Despite this suppression of cooling, an excessively large fraction of galaxies with $\log _{10}\left(M_{*} / \mathrm{M}_{\odot}\right)<10.5$ are star-forming in the AGN-only case. This reflects the large amount of cold gas available (since none is removed by SN feedback) which leads to more massive black holes and to longer gas exhaustion times at given stellar mass, and to much larger black hole and cold gas masses at given halo mass.

The situation is very different when SN feedback is included (right panel). The large hot gas ejection rates in low-mass galaxies result in much lower stellar and gas fractions at given halo mass, and in weak black hole growth for $\log _{10}\left(M_{*} / \mathrm{M}_{\odot}\right) \lesssim 10.5$ corresponding to $\log _{10}\left(M_{200 \mathrm{c}} / \mathrm{M}_{\odot}\right) \lesssim 12$. This produces an almost flat heating-to-cooling ratio in this regime. As the halo mass for which SN ejection becomes ineffective is approached, black hole and hot gas masses become large enough that AGN feedback can prevent all cooling of hot gas. Since SN feedback restricts the cold gas available in all galaxies, the suppression of cooling leads to gas exhaustion and to a clear separation between star forming and quenched galaxies, respectively below and above the cooling dominated/heating dominated transition line. It is clear from Fig. 6 that the stellar mass scale at which this transition occurs evolves only weakly for $z \leqslant 3$ (the solid bars at the bottom of the right panel show significant overlap). This is a consequence of the shape of the relation between heating-to-cooling ratio and stellar mass, in particular, of its 


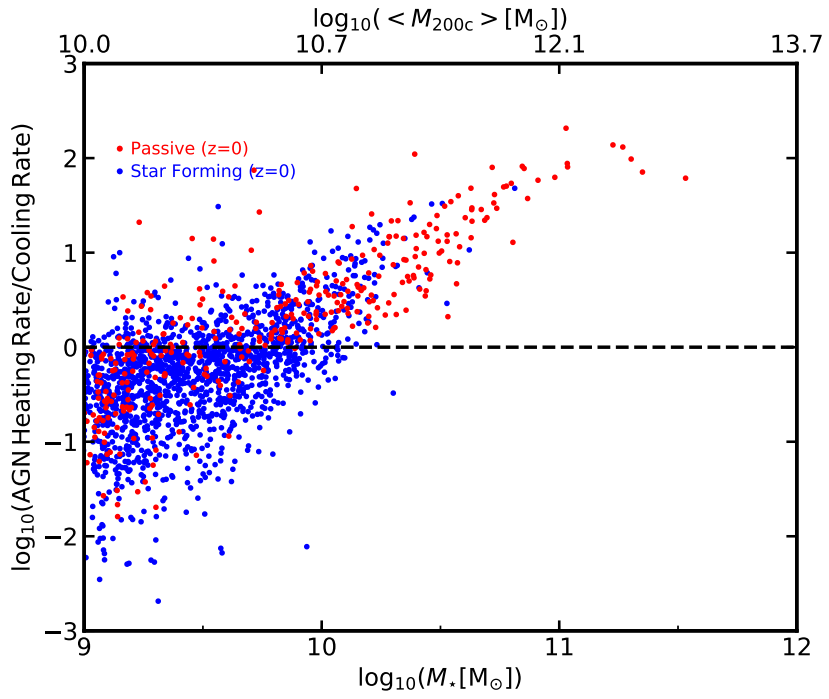

(a) AGN-feedback-only

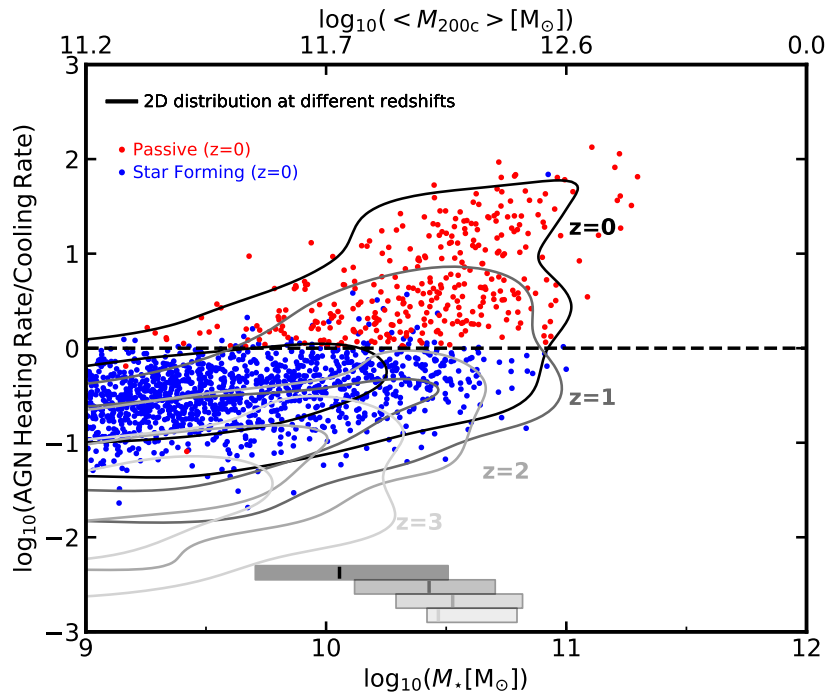

(b) Henriques et al. (2015)

Figure 6. The ratio of maximum AGN radio-mode heating rate to the cooling rate from the hot gas atmosphere as a function of stellar mass for central galaxies in two different models: AGN feedback only (on the left) and the full H15 model (on the right). The coloured dots show the ratio for individual objects at $z=0$ with red symbols representing quenched galaxies and blue symbols representing star-forming galaxies (divided at $\left.\log _{10}\left(s S F R\left[\mathrm{yr}^{-1}\right]\right)=-11.0\right)$. The dashed black line divides galaxies where cooling is fully suppressed (above), from galaxies where it still continues at a reduced rate (below). The solid contours on the right panel show the 2D distribution of galaxies at different redshifts, while the vertical lines and solid bars at the bottom of this panel represent the median and the $16 \%$ to $84 \%$ range of stellar mass for galaxies that just crossed the black dotted line upwards (i.e. were just quenched). From top to bottom the bars refer to redshifts $\mathrm{z}=0,1,2$ and 3 . The top $x$-axes show the average $M_{200 c}$ for a given stellar mass at $z=0$. Since galaxies with $\log _{10}\left(M_{*} / \mathrm{M}_{\odot}\right) \sim 9$ are hosted by haloes with very low mass in the AGN-only model we only show results based on the MRII simulation for this plot.

change in slope at $\log _{10}\left(M_{*} / \mathrm{M}_{\odot}\right) \sim 10.5$ (corresponding to $\left.\log _{10}\left(M_{200 \mathrm{c}} / \mathrm{M}_{\odot}\right) \sim 12\right)$.

\subsection{How important are mergers?}

Mergers play a major role in regulating feedback in our model, because black hole growth occurs primarily through merger-driven accretion of cold interstellar gas. In addition, of course, mergers provide a second channel for galaxies to increase their stellar mass in addition to in situ star formation. In this section we investigate how the relative importance of these two channels is affected by the feedback processes we are investigating.

In Fig. 7 we show the stellar fractions at $z=0$ coming from in situ formation (blue) and from merging satellites (red). The dotted lines show the same quantities but including intra-cluster stars (ICM, hereafter) which originate from tidally disrupted satellites. We do not separate out stars from merger-driven starbursts since this channel is negligible in our model $(\lesssim 3 \%)$ and restrict our analysis to Millennium-II based results in order to correctly resolve properties that strongly depend on merger histories. The behaviour in these plots results from the interplay between the statistics of halo mergers and the halo occupation distribution of galaxies. While the former has no dependence on baryonic processes in our model, the latter varies strongly. Halo merger trees are almost self-similar over the range of masses relevant here, with only a very weak dependence of merger rates on halo mass (approximately $\propto M_{200 c}^{0.1}$, see Fakhouri et al. 2010).

Fig. 2 shows that at low halo mass the stellar mass fraction is large and approximately constant in models without stellar feedback (no-feedback and AGN-only models, two left panels), while when $\mathrm{SN}$ feedback is included (SN-only and H15 models, two right panels), it rises rapidly with halo mass to a peak near $\log _{10}\left(M_{200 c} / \mathrm{M}_{\odot}\right) \sim 12$. In all models the stellar mass fraction decreases at high halo mass (for $\log _{10}\left(M_{200 c} / \mathrm{M}_{\odot}\right) \gtrsim 11.0$ and 12.0 in the cases without and with SN feedback, respectively). The top axes in Fig. 7 show that these halo masses correspond to stellar masses where the fraction of accreted stars starts to rise substantially $\left(\log _{10}\left(M_{*} / \mathrm{M}_{\odot}\right)=10.0\right.$ and 11.0 in the cases without, and with SN feedback, respectively two left and two right panels). At lower mass the fraction of accreted stars drops slowly when SN feedback is absent and very rapidly when it is included, corresponding to the different slopes at low mass in Fig. 2

In detail, we see that in the absence of feedback (left panel), merger contributions to the final stellar mass of galaxies increase slowly as cooling efficiencies drop, but still only amount to $\sim 25 \%$ at $\log _{10}\left(M_{*} / \mathrm{M}_{\odot}\right)=12(\sim 40 \%$ including ICL). For the AGN-only model (second panel from the left) the rise in the merger contribution at high mass is much stronger, corresponding to the steep decline of insitu star formation caused by the AGN-driven suppression of cooling; massive galaxies are almost all quenched by AGN 


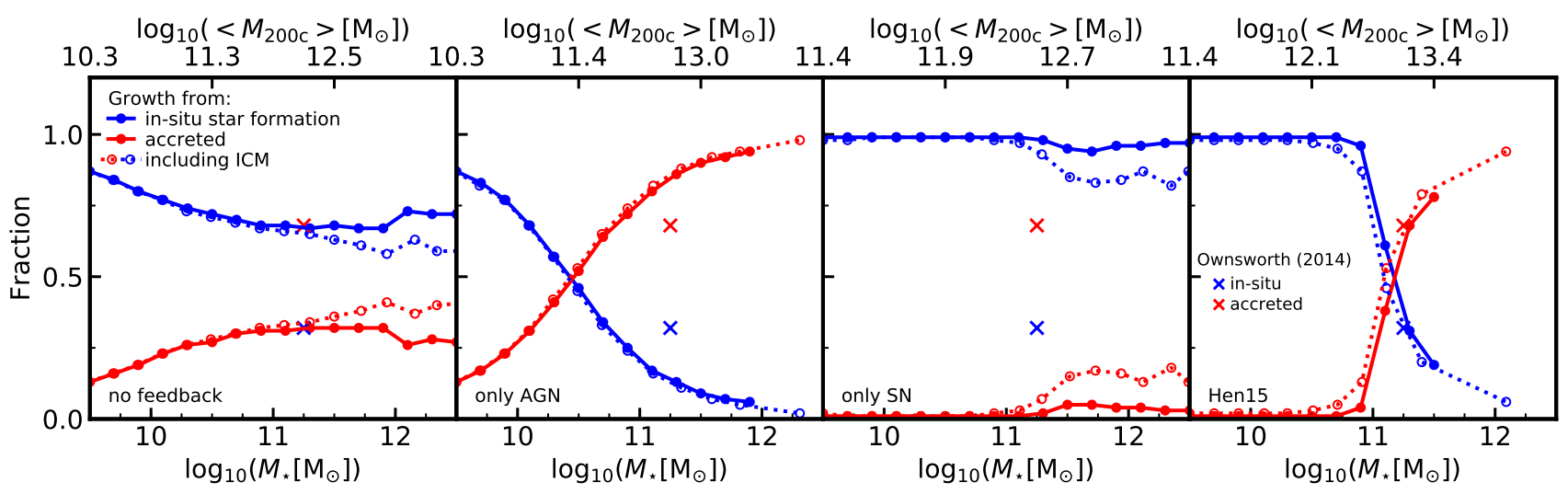

Figure 7. Stellar mass fractions at $z=0$ from in situ star formation (solid blue lines) and from merging satellites (solid red lines) as a function of stellar mass. The dotted lines show the same quantities but including intra-cluster stars. Starbursts are always negligible in our model (contributing $\lesssim 3 \%$ ) and are not shown. From left to right, the panels give results for models with no feedback, with AGN feedback only, with SN feedback only, and with the full Henriques et al. (2015) feedback model. The top $x$-axis in each panel shows the median halo mass, $M_{200 c}$, at given central galaxy stellar mass (without intracluster stars). Note that it differs from panel to panel. This plot is restricted to MR-II based results in order to correctly resolve properties that strongly depend on merger histories. Coloured crosses show observational data from Ownsworth et al. (2014), assuming similar in-situ and accreted fractions below and above $z=3$.

feedback and can only grow by mergers. Furthermore, in the absence of SN feedback, lower mass satellite halos contain relatively massive stellar components which contribute significantly to the central galaxy when they merge. In the case of the SN-only model (second panel from the right), the contribution from mergers is negligible at all masses for the opposite reason; regulation of star formation due to SN winds in low mass satellites means that they contribute relatively few stars to the more massive centrals they merge with. Massive galaxies are almost all star-forming and the in situ contribution is dominant in this model. When both feedback channels are present, mergers contribute a negligible fraction of the stars at low mass, for the same reason as in the SN-only model (the stellar content is reduced in low mass satellite galaxies), and a significant fraction of the stars at high mass, for the same reason as in the AGN-only model (quenched galaxies can only grow through mergers).

In our model, stars accreted from mergers invariably end up residing in a bulge component. Hence, the red lines in Fig. 7 can be read additionally as the fraction of stars in bulges (with the blue lines indicating the fraction of stars in disks). Consequently, in the presence of AGN feedback (second and fourth panels of Fig. 7) high mass galaxies are bulge dominated structures quenched due to AGN feedback. Thus, our model offers an explanation to the strong observational link between bulge-to-total ratio and quenching at fixed stellar mass (e.g. Bluck et al. 2014, Lang et al. 2014 Omand et al. 2014) via AGN feedback (as argued for in Bluck et al. 2016 and Teimoorinia et al. 2016) without requiring quenching to be driven explicitly by morphology as advocated, for example, by Martig et al. (2009).

\section{DISCUSSION}

The complex interplay between the various physical processes included in our model is perhaps best appreciated by studying the evolution of a typical massive galaxy. In

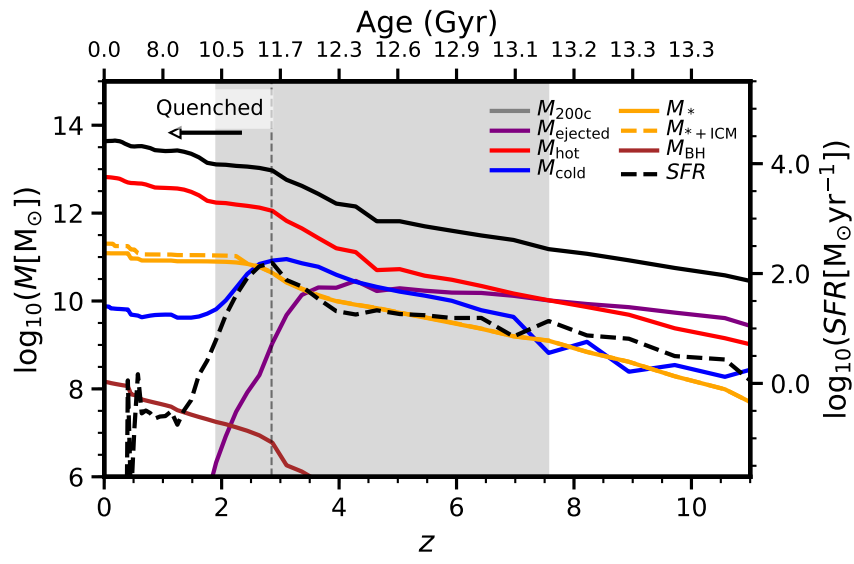

Figure 8. The evolution of various properties as functions of redshift (bottom $x$-axis) or age (top $x$-axis) for a typical massive central galaxy in $\mathrm{H} 15$ model $\left(\log _{10}\left(M_{*} / \mathrm{M}_{\odot}\right) \sim 11.1\right.$ at $\left.z=0\right)$ that quenched at $z \sim 2.0\left(s S F R<(1+z) / 2 t_{\mathrm{H}}\right.$, following Terrazas et al. 2016b). The solid black, purple, blue, red, yellow and brown lines represent the evolution of the masses of the halo, the ejected gas, the cold gas, the hot gas, the stars and the black hole, respectively, with values shown on the left $y$-axis. The dashed yellow line shows the stellar mass including stars assigned to the intracluster light component. The dashed black line shows the evolution of the SFR with values shown on the right $y$-axis. The shaded region highlights the redshift range between the time when the hot component becomes more massive than the external reservoir and that when star formation is quenched. The vertical dashed lines mark the peak of star formation activity.

Fig. 8 we show the growth of the various components of a galaxy which at $z=0$ has stellar mass $\log _{10}\left(M_{*} / \mathrm{M}_{\odot}\right) \sim$ 11.1 and is the central object in a group of total mass $\log _{10}\left(M_{200 c} / \mathrm{M}_{\odot}\right) \sim 13.6$. The solid black, purple, blue, red, yellow and brown lines represent the evolution of the masses 
of the halo, the ejected gas, the cold gas, the hot gas, the stars and the black hole, respectively, with values shown on the left $y$-axis. The dashed yellow line shows the stellar mass including stars assigned to the interstellar light, while the dashed black line shows the evolution of the SFR with values shown on the right $y$-axis. The shaded region highlights the redshift range between the time when the hot component becomes more massive than the external reservoir and that when star formation is quenched. The vertical dashed lines mark the peak of star formation activity

At $z>7$, the halo is small enough to allow supernova feedback to eject large amounts of hot gas and the external reservoir contains most of the baryons. After $z \sim 7$, however, ejection and reincorporation of gas balance out (high redshift boundary of the shaded region) and the mass in the external reservoir remains almost constant until $z \sim 3$ when the halo mass rises above $10^{12} \mathrm{M}_{\odot}$ and ejection ceases. Since the reincorporation time (assumed inversely proportional to $M_{200 c}$ ) has by then dropped below the age of the universe, all ejecta are rapidly reabsorbed by the hot halo. Over the whole period $0 \leqslant z<7$ the hot gas mass increases in step with the halo mass and dominates the total baryon budget of the system.

For $z>7$ the mass in cold gas is similar to that in stars and the SFR is a few $\mathrm{M}_{\odot} / \mathrm{yr}$. However, as SN ejection efficiency decreases and the hot component becomes dominant, the growth of the amount of cold gas accelerates until it exceeds the stellar mass by a factor of several. This induces a steep increase in the SFR reaching values of $\sim$ one hundred $\mathrm{M}_{\odot} / \mathrm{yr}$ (vertical dashed line). A merger at around $z \sim 3.0$ leads to the dumping of $\sim 10^{7} \mathrm{M}_{\odot}$ of cold gas onto the central black hole, which is then massive enough for AGN feedback to shut down further cooling from the hot gas halo. As a result, the cold gas mass and the SFR plummet and the galaxy quenches (low redshift boundary of the shaded region). There is very little growth in the stellar mass of the central galaxy after quenching, since the H15 model assigns the stars from disrupting and merging satellites primarily to the intracluster light component (compare the dashed and solid yellow lines in Fig. 8).

Comparison with Fig. 5 shows that the behaviour of the galaxy of Fig. 8 is typical for its halo mass, and that the same happens to central galaxies of lower mass haloes but at later times. When the halo mass reaches $\sim 10^{12} \mathrm{M}_{\odot}$ (corresponding to $\left.M_{*} \sim 10^{10} \mathrm{M}_{\odot}\right)$, SN stop being able to eject gas from haloes and galaxies experience a phase of increased accretion, intense star formation and accelerated black hole growth. As indicated by the peak in SFR just before quenching in Fig. 8 (dashed black line), this phase is even more dramatic if the galaxy experiences a major merger. For the preferred parameters of the H15 model this occurs at about the same mass at which haloes transition between the rapid cooling ("cold flow") and the static atmosphere ("cooling flow") regimes. The rapid growth of the central black hole then coincides with that of a hot gas atmosphere, resulting in greatly increased AGN feedback. The relatively narrow range in black hole mass at which cooling is offset and galaxies are quenched $\left(M_{\mathrm{BH}} \sim 10^{7} \mathrm{M}_{\odot}\right)$ is reached shortly thereafter, hence also in a narrow and weakly evolving range in halo mass.

When the observed evolution of galaxy abundances and red fractions as a function of stellar mass are used as con- straints, our MCMC sampling of parameter space requires (1) a strong inverse relation between the ability of SN to eject gas from the halo and $V_{\max }$ as well as (2) a direct relation between reincorporation of this gas and $M_{200 c}$. In the hydro simulations of Dubois et al. (2015) and Bower et al. (2017) this behaviour seems to result from the inability of SN winds to penetrate a static hot atmosphere. Independently of the specific modelling implementation, a strong dependence of SN ejection efficiency on halo mass, coupled with an AGN feedback model grounded on black hole growth via cold gas accretion, can easily result in roughly constant characteristic halo and stellar masses for quenching and maximal conversion of baryons into stars. Particularly interesting is the presence of this behaviour in a model where quenching is caused by AGN feedback and not directly linked to halo or stellar mass.

\section{SUMMARY}

The present work aims at understanding the physical origin behind two observational trends: the redshift independence of the characteristic halo and stellar masses at which galaxies quench; and their coincidence with the scale at which baryon conversion into stars maximises.

The same behaviour is seen in the H15 model, where it can be understood as a consequence of galaxies passing through the following three evolutionary phases:

- the stellar and halo masses of central galaxies reach a roughly redshift-independent mass at which supernovae are no longer able to eject material to large radii where it can remain unavailable for reaccretion;

- subsequently, enhanced accretion of hot halo gas onto galaxies produces a relatively large cold gas to halo mass ratio, causing enhanced star formation and enhanced black hole growth

- soon thereafter, the masses of the black hole and of the hot gas halo have grown to the point where AGN feedback stops all further cooling onto the galaxy, leading to quenching as it runs out of fuel, and leaving it with a stellar to halo mass ratio which is close to maximal.

Within such a model, the specific values selected for the 17 parameters allowed to vary in the MCMC chains of H15 are required to obtain consistency with the observed abundance and passive fraction of galaxies as a function of stellar mass over the range $0 \leqslant z \leqslant 3$. As Fig. S9 of H15 shows, the values of all 17 parameters are quite well constrained, so the coincidences and regularities we have focused on in this paper can all be viewed as required by the observational data, at least in the context of models of this type. The actual values obtained for the parameters can then be taken as observational estimates for the efficiencies and the scaling properties of the various cooling, star formation, black hole formation and feedback processes considered. More detailed simulation work is, of course, required to check that the representation of these processes is appropriate, and to understand why the efficiencies and scalings have the values the observations appear to require. 


\section{ACKNOWLEDGEMENTS}

BMBH (ORCID 0000-0002-1392-489X) acknowledges support from a Zwicky Prize fellowship. B.A.T. is supported by the National Science Foundation Graduate Research Fellowship under Grant No. DGE 1256260.

\section{REFERENCES}

Angulo R. E., White S. D. M., Springel V., Henriques B., 2014, MNRAS, 442, 2131

Baldry I. K., Glazebrook K., Brinkmann J., Ivezić Ž., Lupton R. H., Nichol R. C., Szalay A. S., 2004, ApJ, 600, 681

Behroozi P. S., Wechsler R. H., Conroy C., 2013, ApJ, 770, 57

Bell E. F., Monachesi A., Harmsen B., de Jong R. S., Bailin J., Radburn-Smith D. J., D'Souza R., Holwerda B. W., 2017, ApJ, 837, L8

Bell E. F., Wolf C., Meisenheimer K., Rix H.-W., Borch A., Dye S., Kleinheinrich M., Wisotzki L., McIntosh D. H., 2004, ApJ, 608, 752

Birrer S., Lilly S., Amara A., Paranjape A., Refregier A., 2014, ApJ, 793, 12

Bluck A. F. L., Mendel J. T., Ellison S. L., et al., 2016, MNRAS, 462, 2559

Bluck A. F. L., Mendel J. T., Ellison S. L., Moreno J., Simard L., Patton D. R., Starkenburg E., 2014, MNRAS, 441,599

Bogdan A., Lovisari L., Volonteri M., Dubois Y., 2017, ArXiv e-prints

Bower R. G., Benson A. J., Malbon R., Helly J. C., Frenk C. S., Baugh C. M., Cole S., Lacey C. G., 2006, MNRAS, 370,645

Bower R. G., Schaye J., Frenk C. S., Theuns T., Schaller M., Crain R. A., McAlpine S., 2017, MNRAS, 465, 32

Boylan-Kolchin M., Springel V., White S. D. M., Jenkins A., Lemson G., 2009, MNRAS, 398, 1150

Brinchmann J., Charlot S., White S. D. M., Tremonti C., Kauffmann G., Heckman T., Brinkmann J., 2004, MNRAS, 351, 1151

Bundy K., Ellis R. S., Conselice C. J., 2005, ApJ, 625, 621 Croton D. J., Springel V., White S. D. M., De Lucia G., Frenk C. S., Gao L., Jenkins A., Kauffmann G., Navarro J. F., Yoshida N., 2006, MNRAS, 365, 11

Dubois Y., Volonteri M., Silk J., Devriendt J., Slyz A., Teyssier R., 2015, MNRAS, 452, 1502

Faber S. M., Willmer C. N. A., Wolf C., et al., 2007, ApJ, 665,265

Fakhouri O., Ma C.-P., Boylan-Kolchin M., 2010, MNRAS, 406, 2267

Guo Q., White S., Boylan-Kolchin M., De Lucia G., Kauffmann G., Lemson G., Li C., Springel V., Weinmann S., 2011, MNRAS, 413, 101

Guo Q., White S., Li C., Boylan-Kolchin M., 2010, MNRAS, 404, 1111

Henriques B. M. B., White S. D. M., Thomas P. A., Angulo R., Guo Q., Lemson G., Springel V., Overzier R., 2015, MNRAS, 451, 2663

Henriques B. M. B., White S. D. M., Thomas P. A., Angulo R. E., Guo Q., Lemson G., Springel V., 2013, MNRAS, 431,3373
Henriques B. M. B., White S. D. M., Thomas P. A., Angulo R. E., Guo Q., Lemson G., Wang W., 2016, ArXiv e-prints Ilbert O., McCracken H. J., Le Fèvre O., et al., 2013, Astronomy and Astrophysics Supplement Series, 556, A55

Kauffmann G., Haehnelt M., 2000, MNRAS, 311, 576

Kauffmann G., Heckman T. M., Tremonti C., Brinchmann J., Charlot S., White S. D. M., Ridgway S. E., Brinkmann J., Fukugita M., Hall P. B., Ivezić Ž., Richards G. T., Schneider D. P., 2003, MNRAS, 346, 1055

Kauffmann G., Heckman T. M., White S. D. M., et al., 2003, MNRAS, 341, 33

Lang P., Wuyts S., Somerville R. S., et al., 2014, ApJ, 788, 11

Martig M., Bournaud F., Teyssier R., Dekel A., 2009, ApJ, 707,250

Moster B. P., Naab T., White S. D. M., 2012, ArXiv (arxiv:1205.5807)

Moster B. P., Somerville R. S., Maulbetsch C., van den Bosch F. C., Macciò A. V., Naab T., Oser L., 2010, ApJ, 710, 903

Muzzin A., Marchesini D., Stefanon M., et al., 2013, ApJ, 777, 18

Omand C. M. B., Balogh M. L., Poggianti B. M., 2014, MNRAS, 440, 843

Ownsworth J. R., Conselice C. J., Mortlock A., Hartley W. G., Almaini O., Duncan K., Mundy C. J., 2014, MNRAS, 445, 2198

Peng Y., Maiolino R., Cochrane R., 2015, Nat., 521, 192

Peng Y.-j., Lilly S. J., Kovac K., et al., 2010, ApJ, 721, 193

Rodriguez-Puebla A., Primack J. R., Avila-Reese V., Faber S. M., 2017, ArXiv e-prints

Scarlata C., Carollo C. M., Lilly S. J., et al., 2007, ApJ Supp., 172, 494

Springel V., et al., 2005a, MNRAS, 361, 776

Springel V., et al., 2005b, Nat., 435, 629

Teimoorinia H., Bluck A. F. L., Ellison S. L., 2016, MNRAS, 457, 2086

Terrazas B. A., Bell E. F., Woo J., Henriques B. M. B., 2017, ApJ, 844, 170

Terrazas B. A., et al., 2016a, ApJ, 830, L12

Terrazas B. A., et al., 2016b, MNRAS, 459, 1929

Thomas D., Maraston C., Bender R., Mendes de Oliveira C., 2005, ApJ, 621, 673

Tomczak A. R., Quadri R. F., Tran K.-V. H., et al., 2013, ArXiv e-prints

White S. D. M., Frenk C. S., 1991, ApJ, 379, 52 